\title{
Letters
}

\section{A review of the respiratory management of a patient with high level tetraplegia}

This clinical case is of great interest since respiratory failure is the major acute cause of death in traumatic tetraplegic patients, and the study of such patients has added to our knowledge of the physiology and the mechanics of breathing.

We are not given enough information about this 24 year old man:

1. Did he have a normal chest X-ray on admission?

2. What were his blood oxygen and $\mathrm{CO} 2$ levels on admission?

3. What was his respiratory rate?

Immediately after injury, patients with cervical injuries can compensate for a reduced vital capacity by breathing very rapidly with shallow breaths.

The vital capacity must be measured in all positions that the patient is nursed in, since if one leaf of the diaphragm is paralysed, whilst it may support ventilation when the patient is on their back and be adequate when the paralysed leaf is underneath, when they are turned on the side with the paralysed leaf uppermost, this can lead to respiratory embarrassment and death. ${ }^{1}$

Recording these simple parameters can enable the patient to be effectively monitored without recourse to some of the detailed studies alluded to.

I was concerned with these problems when I first started working in spinal injuries in 1956 and found an acute mortality of $38 \%$ in patients with a complete tetraplegia. I initiated various studies to investigate the respiratory problems. $^{2}$

EMG studies have demonstrated in the stage of spinal shock, flaccid paralysis of the intercostal muscles. ${ }^{3}$ At that stage it may be possible, though extremely difficult, to monitor by cutaneous electrodes, the function of the diaphragm. Accurate localisation can be obtained by needle studies or wires but there is a risk of producing a pneumothorax which would be fatal in a tetraplegic patient at this stage.

A systemic study of diaphragmatic function by means of an oesophageal electrode has been performed in the later stages ${ }^{4,5}$ but the electrode needs anchoring with a balloon and, again, in the presence of ileus and a sick patient, is not really justified. They did reveal that when the diaphragm was partially innervated, re-innervation could be accompanied by spasm of the diaphragm. I have made one study in the stage of spinal shock in collaboration with Mike Goldman and Jeremiah Mead with an oesophageal electrode but this cannot be recommended for routine use.

With regard to the increased workload associated with flaccid paralysis of the intercostal muscles referred to by Dr Brown, study of the oxygen consumption of respiratory muscles in established tetraplegic patients when compensatory and hypertrophy of the accessory muscles has occurred, has shown that the oxygen consumption is raised. ${ }^{6}$ I know of no studies in the acute stage.
Under normal circumstances, the respiratory muscles work at optimum efficiency to drive the respiratory system. In these conditions, energy demands will comfortably be exceeded by supply. If the system is inefficient, the energy demands of respiratory muscles will increase, and if they outstrip supply, fatigue will ensue until the body can reduce the load or make adaptive responses. The energy demands of the respiratory muscles increase considerably when the respiratory rate or tidal volume increases above the optimum efficiency. The work of breathing will also be increased if the lungs are stiff and hard to inflate, or the rib cage compliance is decreased.

The rapid breathing alluded to in the acute stage to try and compensate to the reduced vital capacity can lead to exhaustion over a period of, maybe, 2 or 3 days, coupled with the development of oedema in the lungs or pneumonia so the patient becomes exhausted and dies. It is advisable, when the vital capacity is reduced, to support the ventilation by means of a ventilator.

Ledsome and Sharp ${ }^{7}$ made a systematic study of five patients with lesions at $\mathrm{C} 4$ and found results very similar to this patient. They found the average vital capacity in the first week was $1.28 \mathrm{~L}$, which was $24.4 \%$ of predicted (range, 11 to $33 \%$ ). All five patients required ventilator assistance, begun at 1 to 5 days after injury because of severe hypoxaemia or hypercapnia. Two patients with higher FVC (28 and 33\%) were not ventilated initially, but their FVC did decrease before ventilator support was begun. After the initial stage, 2 to 3 weeks, all patients were eventually able to maintain spontaneous ventilation. After 3 months the average FVC was $2.24 \mathrm{~L}(44 \%$ of predicted; range, 18 to $62 \%$ ).

These patients did not have paralysed diaphragms.

In the initial stages of tetraplegia, the chest wall is flaccid and diaphragm contractions may produce paradoxical movement of the chest wall, resulting in lower recording of $\mathrm{VC}$ than actually represented by diaphragm function.

Flaccid paralysis has been demonstrated electromyographically in the acute stage. ${ }^{3}$ Movement studies were carried out in the later stages which showed that the diaphragm can produce a paradoxical movement. ${ }^{8}$ This is due to the fact that the abdominal muscles are paralysed and instead of the contraction of the diaphragm being resisted by intact abdominal muscles, thus causing expansion of the lower rib cage, paradoxical inward sucking of the chest is produced. Naturally the greater the force, the greater the vital capacity; the greater the paradox.

There is variability in the findings and I know of only one study in the acute stage which was followed up over time. ${ }^{9}$ This showed paradox initially. Movements became positive with time. This work needs amplifying.

JR Silver, MB, BS, FRCP Ed \& Lond Fellow of the Institute of Sports Medicine Consultant in Spinal Injuries 


\section{References}

1 Silver JR. Rehabilitation of the Spinally Injured patient. In: Nichols PJR (ed). Rehabilitation Medicine. 2nd edn. Butterworths: London 1980, pp 223-250.

2 Silver JR, Gibbon NOK. Prognosis in Tetraplegia. British Medical Journal 1968; 4: 79-83.

3 Guttmann L, Silver JR. Electromyographic studies on reflex activity of the intercostal and abdominal muscles in cervical cord lesions. Paraplegia 1965; 3: 1-22.

4 Silver JR, Lehr RP. Electromyographic investigation of the diaphragm and intercostal muscles in tetraplegics. Journal of Neurology, Neurosurgery \& Psychiatry 1981; 44: 837-842.

5 Silver JR, Lehr RP. Dyspnoea during generalised spasms in tetraplegic patients. Journal of Neurology, Neurosurgery \& Psychiatry 1981; 44: $842-845$.

6 Silver JR. The oxygen cost of breathing in tetraplegic patients. Paraplegia 1963/4; 1: $204-214$.

7 Ledsome JR, Sharp JM. Pulmonary function in acute cervical cord injury. American Review of Respiratory Disease 1981; 124: $41-44$.

8 Moulton A, Silver JR. Chest movements in patients with traumatic injuries of the cervical cord. Clin Sci 1970; 39: $407-$ 422 .

9 Morgan MDL, Gourlay AR, Silver JR, Williams SJ, Denison DM. Contribution of the rib cage to breathing in tetraplegia. Thorax 1985; 40: $613-617$.

\section{In reply to Dr $\mathbf{J}$ Silver}

The basic information describing the 24-year-old man was deliberately limited to allow the consultants freedom in explaining what they felt were the important parameters in assessing a newly injured SCI individual. Dr Silver presents an interesting discussion and in a comprehensive lengthy review article, these many pieces of information would need to be brought out. However, that was not the intention of this article.

Robert R Menter MD Attending Physician Craig Hospital CNS Medical Group, PC 3425 S Clarkson Englewood CO8011, USA 